\title{
Adjuvant chemotherapy in patients with stage II colon cancer
}

Hyung Jin Kim

Department of Surgery, St. Vincent's Hospital, College of Medicine, The Catholic University of Korea, Suwon, Korea

Article: The efficacy of chemotherapy in the patients with stage II colon cancer associated with number of high-risk factors (Kim MJ, et al. Korean J Clin Oncol 2018;14:116-9)

The purpose of adjuvant chemotherapy after curative resection of colon cancer is eradication of micrometastatic disease, decreasing the recurrence and improving the overall survival. However, chemotherapy may have risk of side effects, and decrease the quality of life. Therefore, the decision making to adding chemotherapy should be made under considering the balance between the risk of recurrence and chemotherapy.

The advantage of adjuvant chemotherapy in stage III colon cancer is well proven by many randomized controlled trials and now it is regarded as a standard treatment [1]. As for the regimen, traditional 5-fluorouracil (5-FU) and leucovorin, capecitabine monotherapy, FOLFOX, or CAPOX are recommended.

However, in stage II colon cancer, if the curative resection was performed, the prognosis is very good and the overall survival after curative resection is reported around $80 \%[2,3]$. The role of adjuvant chemotherapy in stage II colon cancer is minimal and remains an area of great controversy [4]. And which patients will benefit from adjuvant chemotherapy and what chemotherapy to use also remain an area of great controversy. Because patients with stage II colon cancer comprise a heterogeneous combination including stage IIA (pT3N0), stage IIB (pT4aN0), and stage IIC (pT4bN0).

Received: Dec 30, 2018 Accepted: Dec 31, 2018

Correspondence to: Hyung Jin Kim

Department of Surgery, St. Vincent's Hospital, College of Medicine, The

Catholic University of Korea, 93 Jungbu-daero, Paldal-gu, Suwon 16247,

Korea

Tel: +82-31-881-8960, E-mail: hj@catholic.ac.kr

ORCID: Hyung Jin Kim (https://orcid.org/0000-0002-6315-714X)

Copyright (c) 2018 Korean Society of Surgical Oncology

This is an Open Access article distributed under the terms of the Creative Commons Attribution Non-Commercial License (http://creativecommons.org/licenses/by-nc/4.0) which permits unrestricted non-commercial use, distribution, and reproduction in any medium, provided the original work is properly cited.
Current guidelines recommend adjuvant chemotherapy for stage II colon cancer for the patient with high-risk factors for recurrence such as poorly differentiated histology (exclusive of those cancers that are high microsatellite instability [MSI-H]), lymphatic/vascular invasion, bowel obstruction, $<12$ lymph nodes examined, perineural invasion, localized perforation, or close, indeterminate or positive margins.

As for the adding oxaliplatin, according to the subgroup analysis of MOSAIC and NSABP C-07 trial, no significant benefit was found by adding oxaliplatin to 5-FU and leucovorin to stage II colon cancer $[5,6]$. Therefore, considering the potential risk of side effects of oxaliplatin including neuropathy, it is not recommended to adding oxaliplatin in stage II colon cancer patients.

Another factors should be considered is mismatch repair or MSI testing. it should be performed before deciding adjuvant chemotherapy for stage II colon cancer, because MSI-H patients may have a good prognosis and do not benefit from 5-FU adjuvant therapy [7].

The authors tried to find which patient could omit the adjuvant chemotherapy in stage II colon cancer. And they found that the patients with only one high-risk factor for the recurrence may not have significant benefit by adjuvant chemotherapy [8]. However, it should be interpreted cautiously, because to prove a small potential benefit of adjuvant chemotherapy in stage II colon cancer, large population are inevitably needed.

In conclusion, considering all above factors, conversation between clinicians and patients regarding the potential risks and benefits with adjuvant chemotherapy for stage II colon cancers is very important for decision making. And personalized treatment should be performed. 


\section{CONFLICT OF INTEREST}

No potential conflict of interest relevant to this article was reported.

\section{REFERENCES}

1. Boland GM, Chang GJ, Haynes AB, Chiang YJ, Chagpar R, Xing Y, et al. Association between adherence to National Comprehensive Cancer Network treatment guidelines and improved survival in patients with colon cancer. Cancer 2013;119:1593-601.

2. Bockelman C, Engelmann BE, Kaprio T, Hansen TF, Glimelius B. Risk of recurrence in patients with colon cancer stage II and III: a systematic review and meta-analysis of recent literature. Acta Oncol 2015;54:5-16.

3. Gill S, Loprinzi CL, Sargent DJ, Thome SD, Alberts SR, Haller DG, et al. Pooled analysis of fluorouracil-based adjuvant therapy for stage II and III colon cancer: who benefits and by how much? J Clin Oncol 2004;22:1797-806.

4. Quasar Collaborative Group, Gray R, Barnwell J, McConkey C,
Hills RK, Williams NS, et al. Adjuvant chemotherapy versus observation in patients with colorectal cancer: a randomised study. Lancet 2007;370:2020-9.

5. Tournigand C, Andre T, Bonnetain F, Chibaudel B, Lledo G, Hickish T, et al. Adjuvant therapy with fluorouracil and oxaliplatin in stage II and elderly patients (between ages 70 and 75 years) with colon cancer: subgroup analyses of the Multicenter International Study of Oxaliplatin, Fluorouracil, and Leucovorin in the Adjuvant Treatment of Colon Cancer trial. J Clin Oncol 2012;30:3353-60.

6. Kuebler JP, Wieand HS, O'Connell MJ, Smith RE, Colangelo LH, Yothers G, et al. Oxaliplatin combined with weekly bolus fluorouracil and leucovorin as surgical adjuvant chemotherapy for stage II and III colon cancer: results from NSABP C-07. J Clin Oncol 2007;25:2198-204.

7. $\mathrm{Ng} \mathrm{K}$, Schrag D. Microsatellite instability and adjuvant fluorouracil chemotherapy: a mismatch? J Clin Oncol 2010;28:3207-10.

8. Kim MJ, Baek S, Ko S. The efficacy of chemotherapy in the patients with stage II colon cancer associated with number of high-risk factors. Korean J Clin Oncol 2018;14:116-9. 